\title{
Alkaline Stability of Quaternized Diels-Alder Polyphenylenes
}

Eun Joo Park, ${ }^{a}$ Sandip Maurya, ${ }^{a}$ Michael R. Hibbs, ${ }^{\mathrm{b}}$ Cy H. Fujimoto,,${ }^{\mathrm{b}}{ }^{*}$ Klaus-Dieter Kreuer, ${ }^{\mathrm{c}}$ and Yu Seung Kimª,*

${ }^{a}$ MPA-11, Los Alamos National Laboratory, Los Alamos, New Mexico 87545, USA

b Organic Materials Science, Sandia National Laboratories, Albuquerque, New Mexico 87185, USA

${ }^{c}$ Max-Planck-Institut für Festkörperforschung, Heisenbergstr 1, D-70569 Stuttgart, Germany

*chfujim@sandia.gov; yskim@lanl.gov 


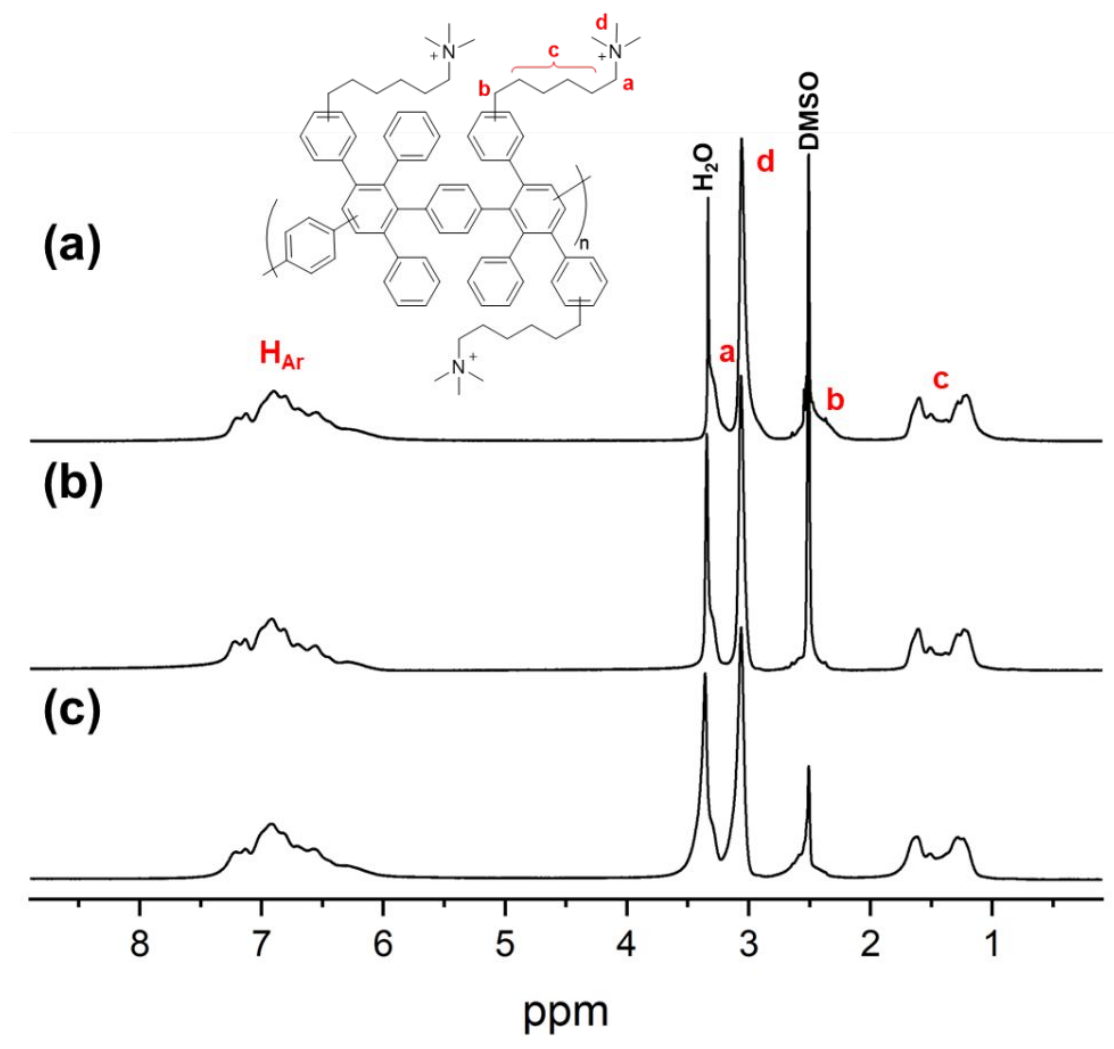

Figure S1. ${ }^{1} \mathrm{H}$ NMR spectra of (a) HTMA DAPP, (b) HTMA DAPP after the alkaline treatment in 4 $\mathrm{M} \mathrm{NaOH}$ at $80{ }^{\circ} \mathrm{C}$ for $500 \mathrm{~h}$ and (c) after the alkaline treatment in $4 \mathrm{M} \mathrm{NaOH}$ at $80{ }^{\circ} \mathrm{C}$ for $1,000 \mathrm{~h}$ in DMSO-d $\mathrm{d}_{6}$. 
Table S1. AEM properties after alkaline stability test.

\begin{tabular}{|c|c|c|c|c|c|c|c|c|c|}
\hline \multicolumn{3}{|c|}{ Sample } & \multirow{2}{*}{ Solubility $^{a}$} & \multirow{2}{*}{$\begin{array}{c}\text { IEC } \\
(\mathrm{meq} . / \mathrm{g})^{b}\end{array}$} & \multirow{2}{*}{$\begin{array}{c}\text { IEC } \\
(\mathrm{meq} . / \mathrm{g})^{c}\end{array}$} & \multirow{2}{*}{$\begin{array}{l}\text { Water } \\
\text { uptake } \\
(\%)^{d}\end{array}$} & \multirow{2}{*}{$\begin{array}{c}\text { Swelling } \\
\text { degree } \\
(\%)^{d}\end{array}$} & \multicolumn{2}{|c|}{$\begin{array}{l}\mathrm{OH}^{-} \text {conductivity } \\
(\mathrm{mS} / \mathrm{cm})\end{array}$} \\
\hline $\begin{array}{l}\text { Temp. } \\
\left({ }^{\circ} \mathrm{C}\right)\end{array}$ & $\begin{array}{c}{[\mathrm{NaOH}]} \\
(\mathrm{M})\end{array}$ & $\begin{array}{c}\text { Test } \\
\text { hours } \\
(\mathrm{h})\end{array}$ & & & & & & $30^{\circ} \mathrm{C}$ & $80^{\circ} \mathrm{C}$ \\
\hline \multicolumn{3}{|c|}{ HTMA DAPP } & Soluble & 2.59 & 2.41 & 58 & 17 & 35 & 119 \\
\hline 80 & 4 & 500 & Soluble & 2.42 & 2.32 & 53 & -- & 29 & 90 \\
\hline 80 & 4 & 1000 & Soluble & 2.32 & 2.31 & 52 & -- & 27 & 88 \\
\hline 80 & 4 & 3000 & Insoluble & 2.36 & -- & 40 & -- & 32 & 73 \\
\hline 120 & 4 & 2 & Soluble & 2.56 & 2.44 & 55 & 17 & 36 & 84 \\
\hline 120 & 4 & 72 & Insoluble & 1.74 & -- & 30 & 10 & 22 & 61 \\
\hline 120 & 8 & 2 & Insoluble & 2.49 & -- & 37 & 11 & 27 & 76 \\
\hline 120 & 8 & 24 & Insoluble & 2.08 & -- & 32 & 10 & 22 & 66 \\
\hline 120 & 8 & 48 & Insoluble & 1.59 & -- & 26 & 7 & 20 & 56 \\
\hline 120 & 8 & 72 & Insoluble & 1.46 & -- & 25 & 6 & 20 & 53 \\
\hline 160 & 4 & 2 & Insoluble & 1.76 & -- & 30 & 11 & 23 & 63 \\
\hline 160 & 4 & 72 & Insoluble & 0.56 & -- & 4 & 0 & 8 & 20 \\
\hline 160 & 8 & 2 & Insoluble & 1.35 & -- & 9 & 5 & 16 & 48 \\
\hline 160 & 8 & 24 & Insoluble & 0.98 & -- & 3 & 0 & 10 & 35 \\
\hline 160 & 8 & 48 & Insoluble & 0.76 & -- & 0.7 & 0 & 8 & 29 \\
\hline 160 & 8 & 72 & Insoluble & 0.52 & -- & 0 & 0 & 4 & 24 \\
\hline
\end{tabular}

${ }^{a}$ Measured at $2.5 \mathrm{w} / \mathrm{v} \%$ in DMSO and DMAc. ${ }^{b}$ The average IEC value of two samples determined by Mohr titration. ${ }^{c}$ The calculated value from the ${ }^{1} \mathrm{H}$ NMR spectra. ${ }^{d}$ The average value from two samples. The membranes in $\mathrm{OH}^{-}$form were immersed in water at room temperature for $24 \mathrm{~h}$. ${ }^{e}$ Measured in deionized water.

Table S2. AEM properties after the additional alkaline stability test.

\begin{tabular}{|c|c|c|c|c|c|c|c|c|c|c|}
\hline \multirow{3}{*}{$\begin{array}{l}\text { Temp. } \\
\left({ }^{\circ} \mathrm{C}\right)\end{array}$} & \multicolumn{2}{|l|}{ Sample } & \multicolumn{3}{|c|}{$\begin{array}{l}\text { Initial membrane } \\
\text { properties }\end{array}$} & \multicolumn{2}{|c|}{$\begin{array}{c}500 \mathrm{~h}, \\
4 \mathrm{M} \mathrm{NaOH}, 80^{\circ} \mathrm{C}\end{array}$} & \multicolumn{3}{|c|}{$\begin{array}{c}1000 \mathrm{~h}, \\
4 \mathrm{M} \mathrm{NaOH}, 80^{\circ} \mathrm{C}\end{array}$} \\
\hline & \multirow{2}{*}{$\begin{array}{l}{[\mathrm{NaOH}]} \\
(\mathrm{M})\end{array}$} & \multirow[t]{2}{*}{$\begin{array}{c}\text { Test } \\
\text { hours }(\mathrm{h}) \text { - }\end{array}$} & \multicolumn{2}{|c|}{$\begin{array}{l}\mathrm{OH}^{-} \text {conductivity } \\
\quad(\mathrm{mS} / \mathrm{cm})\end{array}$} & \multirow{2}{*}{$\begin{array}{c}\text { IEC } \\
\text { (meq./ } \\
\text { g) })^{a}\end{array}$} & \multicolumn{2}{|c|}{$\begin{array}{l}\mathrm{OH}^{-} \text {conductivity } \\
(\mathrm{mS} / \mathrm{cm})\end{array}$} & \multicolumn{2}{|c|}{$\begin{array}{c}\mathrm{OH}^{-} \\
\text {conductivity } \\
(\mathrm{mS} / \mathrm{cm})\end{array}$} & \multirow{2}{*}{$\begin{array}{r}\text { IEC } \\
(\mathrm{meq} \\
/ \mathrm{g})^{a}\end{array}$} \\
\hline & & & $30^{\circ} \mathrm{C}$ & $80^{\circ} \mathrm{C}$ & & $30^{\circ} \mathrm{C}$ & $80^{\circ} \mathrm{C}$ & $30^{\circ} \mathrm{C}$ & $80^{\circ} \mathrm{C}$ & \\
\hline 120 & 8 & 2 & 27 & 76 & 2.49 & 29 & 79 & 26 & 76 & 2.27 \\
\hline 160 & 8 & 2 & 16 & 48 & 1.35 & 19 & 48 & 16 & 47 & 1.28 \\
\hline
\end{tabular}

${ }^{a}$ The average IEC value of two samples determined by Mohr titration. 


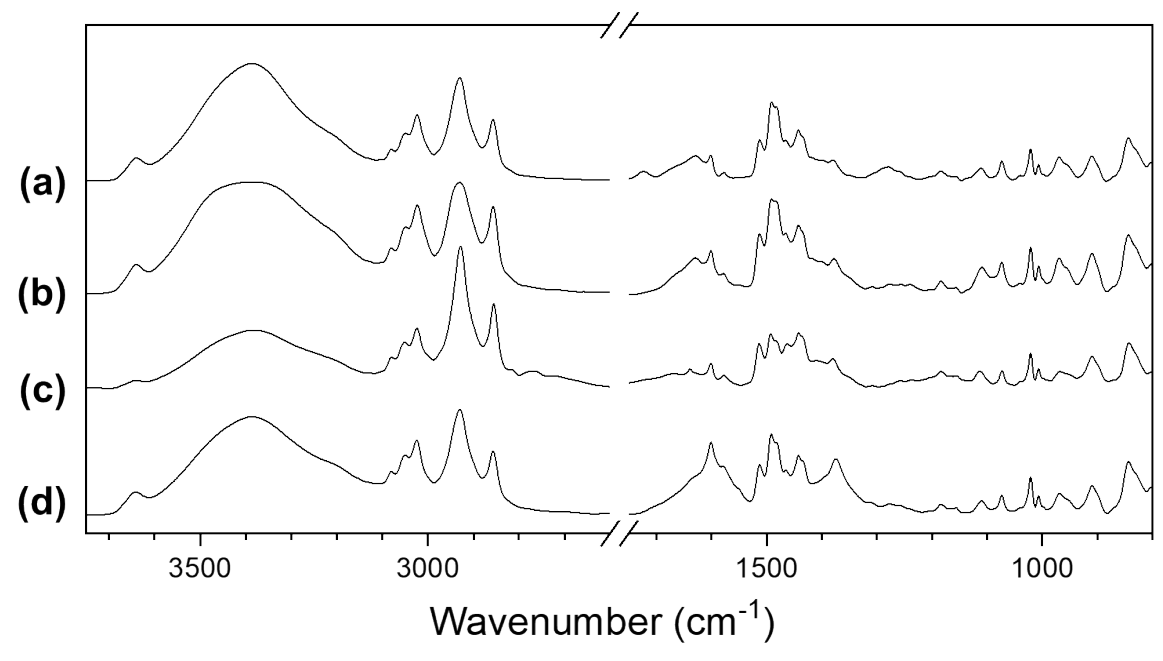

Figure S2. FTIR spectra of HTMA DAPP (a) before the alkaline stability test and after the 2 h-ASTs of (b) $120^{\circ} \mathrm{C}, 8 \mathrm{M} \mathrm{NaOH}$, (c) $160^{\circ} \mathrm{C}, 8 \mathrm{M} \mathrm{NaOH}$, and (d) after the 3,000 h-test at $80^{\circ} \mathrm{C}, 4 \mathrm{M} \mathrm{NaOH}$.

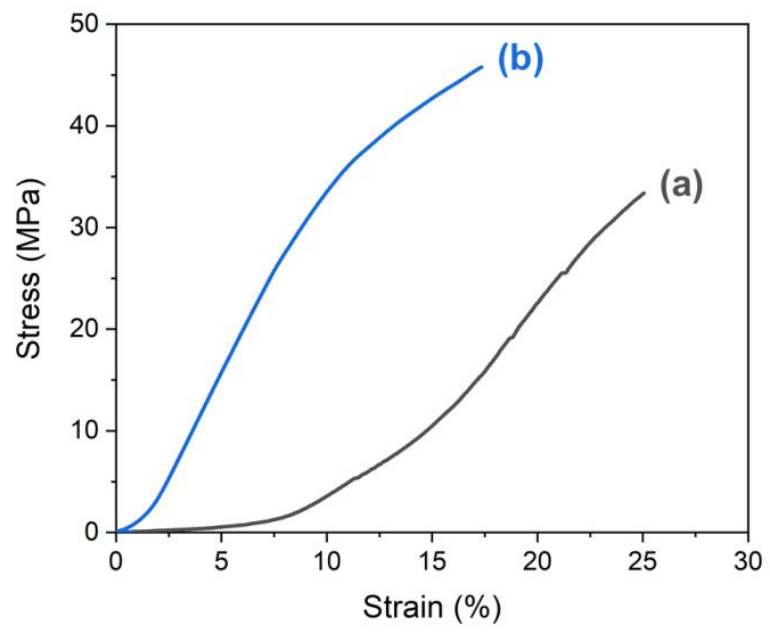

Figure S3. Stress-strain curves of HTMA DAPP $\left(\mathrm{OH}^{-}\right.$form) at $30{ }^{\circ} \mathrm{C}, 0 \% \mathrm{RH}$ (a) before and (b) after $3,000 \mathrm{~h}$ alkaline stability test at $80^{\circ} \mathrm{C}$ in $4 \mathrm{M} \mathrm{NaOH}$. 
Table S3. Membrane properties of AEMs after low RH tests.

\begin{tabular}{|c|c|c|c|c|c|c|c|}
\hline \multirow{2}{*}{\multicolumn{2}{|c|}{$\begin{array}{c}\text { Sample } \\
\text { Temp. }\left({ }^{\circ} \mathrm{C}\right) \text { Test hours }(\mathrm{h})\end{array}$}} & \multirow{2}{*}{ Solubility $^{a}$} & \multirow{2}{*}{$\begin{array}{c}\text { IEC } \\
(\text { meq. } / \mathrm{g})^{b}\end{array}$} & \multirow{2}{*}{$\begin{array}{c}\text { Water } \\
\text { uptake } \\
(\%)^{c}\end{array}$} & \multirow{2}{*}{$\begin{array}{c}\text { Swelling } \\
\text { degree } \\
(\%)^{c}\end{array}$} & \multicolumn{2}{|c|}{$\begin{array}{c}\mathrm{OH}^{-} \text {conductivity } \\
(\mathrm{mS} / \mathrm{cm})\end{array}$} \\
\hline & & & & & & $30^{\circ} \mathrm{C}$ & $80^{\circ} \mathrm{C}$ \\
\hline \multicolumn{2}{|c|}{ Pristine HTMA DAPP } & Soluble & 2.59 & 58 & 17 & 35 & 119 \\
\hline 100 & 24 & Insoluble & 1.20 & 13 & 3 & 16 & 27 \\
\hline 100 & 60 & Insoluble & 0.80 & 10 & 1 & 12 & 22 \\
\hline 100 & 150 & Insoluble & 0.63 & 4 & 0 & 11 & 21 \\
\hline
\end{tabular}

${ }^{a}$ Measured at $2.5 \mathrm{w} / \mathrm{v} \%$ in DMSO and DMAc. ${ }^{b}$ The average IEC value of two samples determined by Mohr titration. ${ }^{c}$ The average value from two samples. The membranes in $\mathrm{OH}^{-}$form were immersed in water at room temperature for $24 \mathrm{~h} .{ }^{e}$ Measured in deionized water.

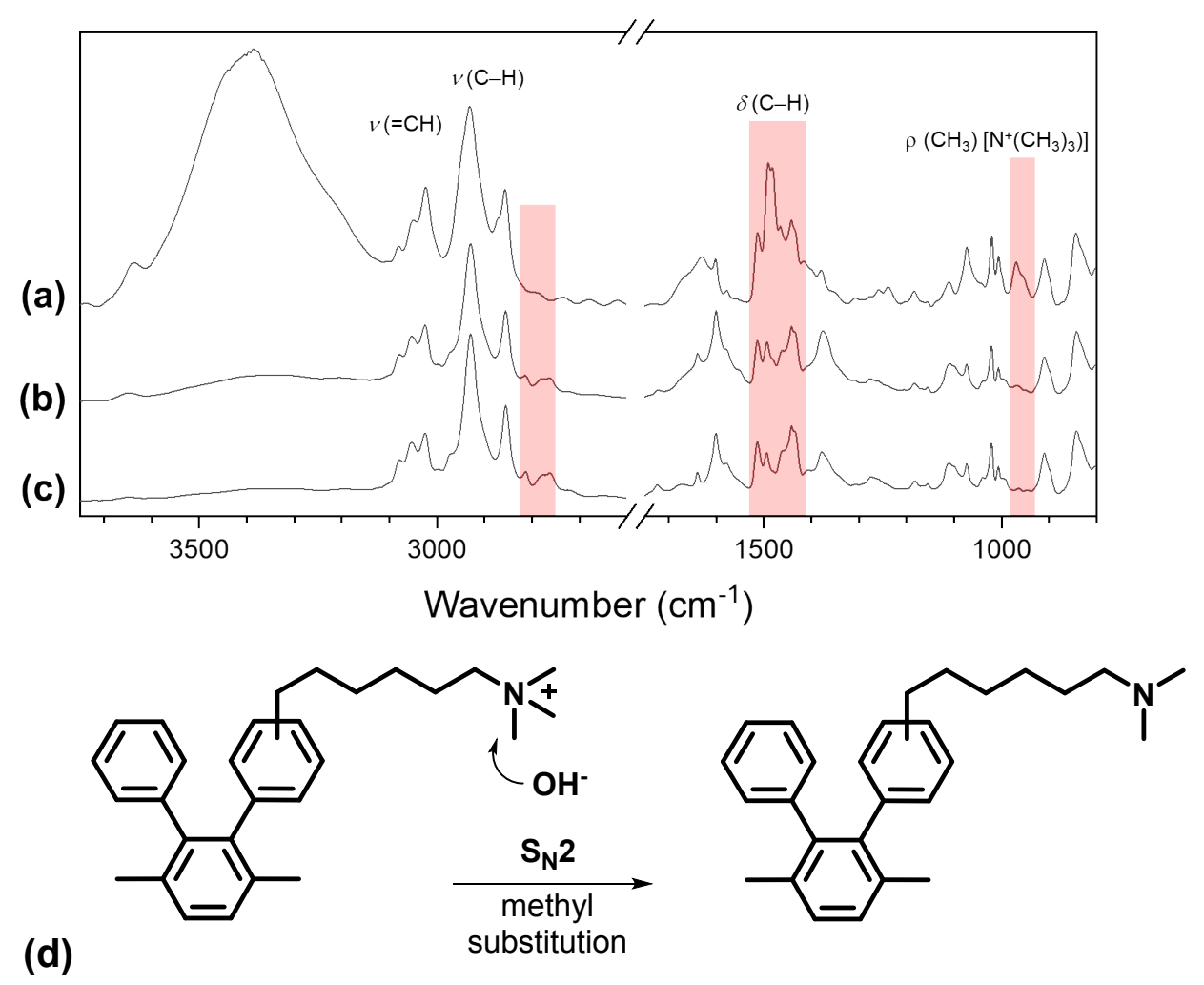

Figure S4. FTIR spectra of HTMA DAPP (a) before and after the low RH ASTs at $100{ }^{\circ} \mathrm{C}$ (b) for $24 \mathrm{~h}$ and (c) for $150 \mathrm{~h}$. (d) Degradation mechanism of quaternary ammonium into tertiary amine via methyl substitution. 


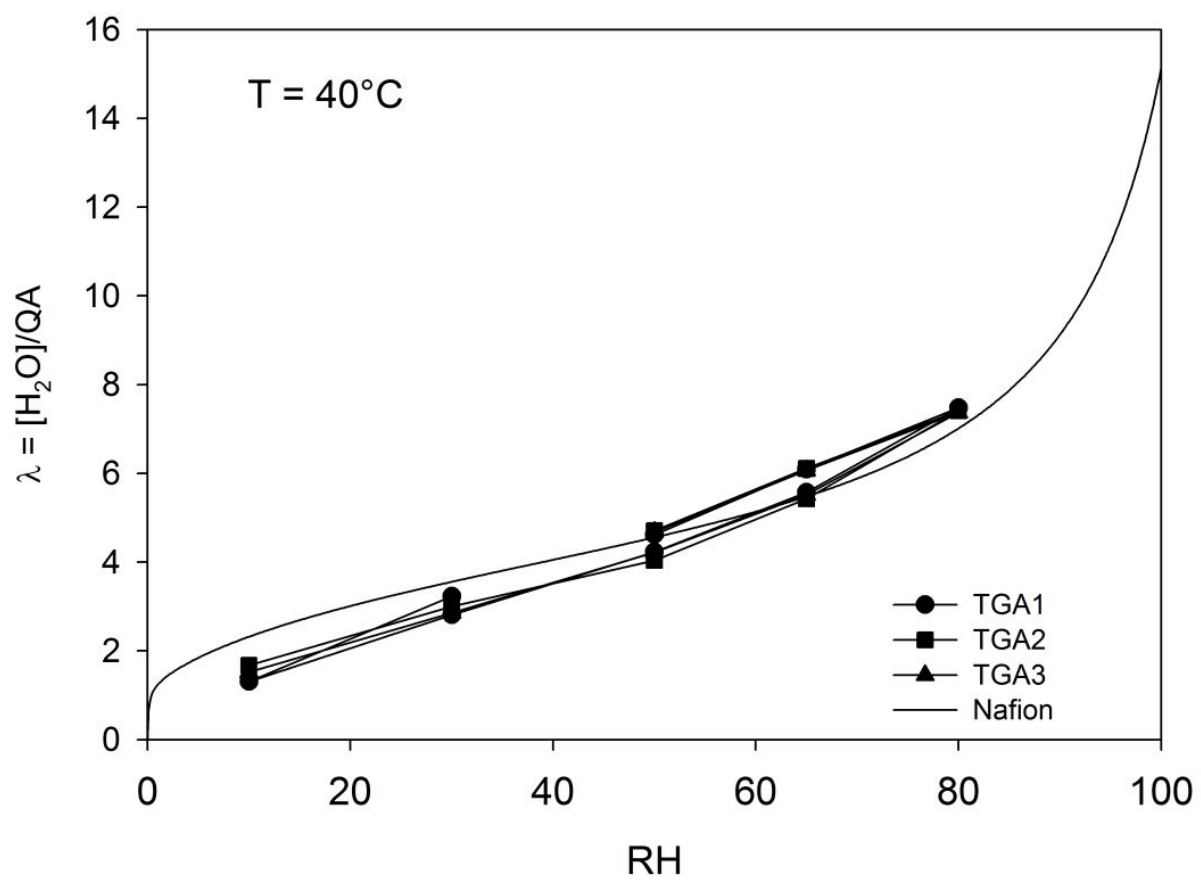

Figure S5. Hydration isotherm of HTMA DAPP (TGA 1-3) compared to the hydration isotherm of Nafion for membrane hydration number $\lambda$ of HTMA DAPP at $\mathrm{T}=40^{\circ} \mathrm{C}$. 